\title{
Optimal Polarization Conversion in Coupled Dimer Plasmonic Nanoantennas for Metasurfaces
}

Leo-Jay Black ${ }^{1,3)}$, Yudong Wang ${ }^{1,2)}$, C. H. de Groot ${ }^{2)}$, Arnaud Arbouet ${ }^{3)^{*}}$, Otto L. Muskens $1)^{*}$

1) Physics and Astronomy, Faculty of Physical Sciences and Engineering, University of Southampton, Highfield, Southampton SO17 1BJ, United Kingdom

2) Nano Group, Faculty of Physical Sciences and Engineering, University of Southampton, Highfield, Southampton SO17 1BJ, United Kingdom

3) CEMES, UPR 8011, CNRS-Université de Toulouse, 29 Rue Jeanne Marvig, BP 94347, F31055 Toulouse, France

KEYWORDS: Plasmonic Nanoantennas, Polarization Conversion, Phase Discontinuity, Metamaterials, Metasurfaces, Green Dyadic Method

ABSTRACT: We demonstrate that polarization conversion in coupled dimer antennas, used in phase discontinuity metasurfaces, can be tuned by careful design. By controlling the gap width, a strong variation of the coupling strength and polarization conversion is found between capacitively and conductively coupled antennas. A theoretical two-oscillator model is proposed which shows a universal scaling of the degree of polarization conversion with the energy splitting of the symmetric and anti-symmetric modes supported by the antennas. Using single antenna spectroscopy, we find good agreement for the scaling of mode splitting and polarization conversion with gap width over the range from capacitive to conductive 
coupling. Next to linear polarization conversion, we demonstrate single-antenna linear to circular polarization conversion. Our results provide strategies for phase-discontinuity metasurfaces and ultracompact polarization optics.

There is an increasing interest in using nanoscale plasmonic devices to control the polarization and propagation states of light. Plasmonic metamaterials with strong birefringence and chirality have been demonstrated, with applications in tunable waveplates and optical biosensors. ${ }^{1,2}$ Recently, plasmonic metasurfaces containing two dimensional phase gradients have been developed which allow imprinting arbitrary phase patterns onto a propagating beam. ${ }^{3}$ Such phase-discontinuity surfaces have already achieved a variety of optical components including lenses, spiral waveplates, and 3D holograms. ${ }^{4-10}$

Among a variety of designs, $\mathrm{V}$-shaped antennas are of particular interest for their ability to support a set of orthogonal eigenmodes with a variable phase delay depending on the apex angle. As a special case of the V-antenna, the L-shaped antenna has an orthogonal orientation of the two arms. The properties of connected L-shaped antennas have been investigated in a number of experimental and theoretical works. ${ }^{3,11-17}$ L-shaped nanoantennas have particularly received interest for their noncentrosymmetric properties in the generation of second harmonic generation, ${ }^{18}$ in polarization conversion and birefringence. ${ }^{12,15,16}$ The above studies all considered connected antenna designs; disconnected nanorod dimers in an orthogonal geometry were explored in Refs. 19-21. Similar to the case of aligned nanoparticle dimers, ${ }^{22,23}$ the nature of the interactions between disconnected and connected rods is qualitatively different. While the resonances of the disconnected particles are coupled through electrostatic, capacitive interactions, the connected rods are governed by chargetransfer plasmon modes. For connected structures, the modes are therefore directly associated with the symmetry of the structure, and are relatively insensitive to the properties of the surrounding environment. For disconnected structures, polarization conversion is mediated 
through the plasmonic near-field coupling between electrically isolated nanoparticles. In this case, polarization conversion can be a sensitive measure of the inter-particle coupling strength depending on the properties and loading of the nanogap, which could provide new means for active control and sensing. ${ }^{24}$ Such orthogonal near-field couplings exist in many nanostructures, including e.g. dolmen antennas and stacked three-dimensional plasmonic rulers. ${ }^{25}$ However systematic studies of such structures generally considered the polarization conserving states only.

In this work, we explore the polarization conversion in antenna designs consisting of two perpendicular nanorods over the entire regime from capacitive coupling to the conductive coupling regime. Building on a simple two-oscillator model, we show that the polarization conversion is governed by the relative mode splitting, independent of the coupling regime, which is sensitively tuned through the interparticle gap. This picture is supported by extensive electrodynamical simulations based on the 3D-Green Dyadic Method. Spectroscopy performed on individual nanoantennas confirms that precise control of the antenna design allows us to go beyond previous investigations and achieve different regimes of coupling strength and spectral characteristics.

\section{Results and Discussion}

Analytical model. The optical anisotropy already demonstrated in L-shaped gold antenna arrays has been interpreted in terms of interferences between the radiated waves emitted by two orthogonal surface plasmon modes excited in each individual nanostructure. ${ }^{12,15,16}$ Disconnected nanorod dimers are another example of nanosystems supporting a pair of orthogonal plasmon modes with a spectral splitting governed by the gap between the two arms. ${ }^{19}$ More generally, any nano-object supporting two orthogonal modes will have polarization properties similar to those of L-shaped antennas. ${ }^{26,27}$ Indeed, let us consider a 
nanostructure supporting two surface plasmon modes A and B polarized along orthogonal directions $\vec{e}_{A}$ and $\vec{e}_{B}$. These eigenmodes can be represented by two independent oscillators characterized by complex polarizabilities $\alpha_{a}$ and $\alpha_{b}$. Assuming a Lorentzian lineshape, $\alpha_{a}$ for instance can be written as:

$$
\alpha_{a}=\frac{\alpha_{0 a} \omega_{0 a}^{2}}{\omega_{0 a}^{2}-\omega\left(\omega-i \Gamma_{a}\right)}
$$

Here $\omega$ denotes the frequency, $\omega_{0 \mathrm{a}}$ is the resonance frequency and $\Gamma_{\mathrm{a}}$ the damping of the oscillator. Figure 1a and $\mathrm{b}$ respectively show the amplitude and phase of the polarizabilities associated with these two modes. For simplicity, we assume that all resonance parameters are the same, except the angular frequency (the validity of this assumption will be confirmed in the following). An incident optical wave polarized along $X$ denoted $\vec{E}_{0}=E_{0} \vec{e}_{X}$ with $\vec{e}_{X}=$ $\left(\vec{e}_{A}+\vec{e}_{B}\right) / \sqrt{2}$ the direction of the electric (E-)field, will induce a net dipole moment on the nanostructure, which is the sum of the contributions of modes A and B.

In the following, we show that it is possible in specific conditions to generate efficiently a scattered wave polarized along the cross-polarized direction $\vec{e}_{Y}=\left(\vec{e}_{A}-\vec{e}_{B}\right) / \sqrt{2}$. The total dipole induced on the nanostructure can be written as:

$\vec{P}_{T}=\vec{P}_{A}+\vec{P}_{B}=\alpha_{a}\left(\vec{E}_{0} \cdot \vec{e}_{A}\right) \vec{e}_{A}+\alpha_{b}\left(\vec{E}_{0} \cdot \vec{e}_{B}\right) \vec{e}_{B}$

The total dipole moment has components along the $\mathrm{X}$ - and Y-directions given by $P_{T, X}=\frac{E_{0}}{2}\left(\alpha_{a}+\alpha_{b}\right)$ and $P_{T, Y}=\frac{E_{0}}{2}\left(\alpha_{a}-\alpha_{b}\right)$. As a first approximation, we can consider that the intensities of the radiated waves polarized along $\mathrm{X}$ (resp. Y) are proportional to the square of the modulus of the total dipole component along X (resp. Y). The intensities and phases of the scattered light for input polarization along $\mathrm{X}$ and with output polarizations along $\mathrm{X}$ and $\mathrm{Y}$ are shown in Figure 1a,b. For the intensities we use the naming convention of $\sigma_{\mathrm{XX}}$ for both 
input and detection polarizations along $\mathrm{X}$ and $\sigma_{\mathrm{XY}}$ for input along $\mathrm{X}$ and detection along $\mathrm{Y}$. These correspond to diagonal and off-diagonal elements of a scattering matrix:

$\sigma_{X X} \propto\left|P_{T, X}\right|^{2}=\left|\frac{\alpha_{a}+\alpha_{b}}{2}\right|^{2}$

$\sigma_{X Y} \propto\left|P_{T, Y}\right|^{2}=\left|\frac{\alpha_{a}-\alpha_{b}}{2}\right|^{2}$

We see in the example of Figure 1 that outside the spectral range between the two optical resonances, the oscillations of modes $\mathrm{A}$ and $\mathrm{B}$ are in-phase whereas they are dephased for frequencies lying between the frequencies of the eigenmodes. The exact dephasing actually depends on the spectral splitting between the two resonances. For very large splittings, it is close to $\pi$. In this case, an incident wave polarized along $X$ will induce two dipole moments with opposite phases along $\mathrm{A}$ and $\mathrm{B}$ and the resulting interference will be polarized along Y. ${ }^{12,16}$ However, for very large splittings, modes A and B are spectrally separated and it is not possible to efficiently excite both modes. This results in a weak scattering along Y. For decreasing splittings, the dephasing between modes A and B decreases but it becomes easier to excite both modes simultaneously as they start to overlap. Therefore, the wavelength of maximum cross-polarized intensity lies between modes A and B where both efficient in- and out-coupling of the optical excitation and the required phase relation between the induced oscillations can be simultaneously obtained. Interestingly, the phase of $\mathrm{P}_{\mathrm{T}, \mathrm{Y}}$ spans a much larger range than $\mathrm{P}_{\mathrm{T}, \mathrm{X}}$. As discussed by Kats et al., ${ }^{16}$ the larger phase range of the crosspolarized light in the case of a system of two coupled oscillators compared to the single oscillator case results from the minus sign in the expression of $\sigma_{\mathrm{XY}}$.

Similar to projection on the $\mathrm{X}-\mathrm{Y}$ linear polarization basis, we can project the induced dipole moment onto the basis for left-handed (LCP) and right-handed (RCP) circular polarization states $\vec{e}_{L C P}=\left(\vec{e}_{X}+i \vec{e}_{Y}\right) / \sqrt{2}$ and $\vec{e}_{R C P}=\left(\vec{e}_{X}-i \vec{e}_{Y}\right) / \sqrt{2}$. The circular polarization components of the total induced dipole moment follow as 
$P_{T, L C P}=\frac{E_{0}}{2 \sqrt{2}}\left[(1+i) \alpha_{a}+(1-i) \alpha_{b}\right]$

$P_{T, R C P}=\frac{E_{0}}{2 \sqrt{2}}\left[(1-i) \alpha_{a}+(1+i) \alpha_{b}\right]$

Perfect circular polarization in the output is obtained for the case that $\alpha_{a}$ and $\alpha_{b}$ are of equal magnitude but out of phase by $\pi / 2$, i.e. $\alpha_{a}=-i \alpha_{b}$, so that $P_{T, R C P}$ completely cancels and the induced dipole moment is fully left-hand circular polarized.

Of interest also is the conversion between two circular polarization states. In this case, the total induced dipole moment is given by

$\vec{P}_{T}=\frac{E_{0}}{2 \sqrt{2}}\left(\alpha_{a}-\alpha_{b}\right)(1+i) \vec{e}_{L C P}+\frac{E_{0}}{2 \sqrt{2}}\left(\alpha_{a}+\alpha_{b}\right)(1-i) \vec{e}_{R C P}$

for LCP input. Therefore, we recover amplitudes for LCP/RCP output which are similar to Xlinear/Y-linear polarization and have the same polarization conversion from LCP to RCP and vice versa as from $\mathrm{X}$ to $\mathrm{Y}$. Thus, the antenna structures are equally suitable for circular as for linear polarization conversion.

Comparison with numerical model calculations. To provide a deeper insight into the polarization conversion in connected or disconnected nanorod dimer antennas, we have performed systematic numerical simulations using the 3D-Green Dyadic Method. ${ }^{28}$ This method provides the electric field distribution inside the investigated nanostructure from a unique generalized field propagator expressed in direct space. The latter is computed from a numerical procedure which relies on a volume discretization of the nanostructure and rigorously takes into account the dielectric properties of substrate and environment (see Methods). The simulated spectra, presented in Figure 2, cover the range from capacitively to conductively coupled antennas. A positive gap is defined as the shortest distance between the end cap edges of the two antennas, while a negative gap indicates the same for the overlapping antennas as is illustrated in the inset of Figure 2. A gap of -90nm corresponds to 
an L-shaped antenna with rounded corner. The mode at $0.65 \mu \mathrm{m}$ wavelength corresponds to the transverse resonance of the nanorods, which does not show a marked hybridization.

The longitudinal modes for the orthogonal nanorod dimers, appearing at wavelengths above $1.0 \mu \mathrm{m}$ in Figure 2, follow the plasmon hybridization trends known for linear nanoparticle dimers. ${ }^{22,23}$ For small positive gaps, capacitive coupling results in a redshift of the bonding mode. Compared to linear dimers, where destructive interference of the dipoles results in a dark anti-bonding mode, ${ }^{23}$ the orthogonal dipoles do not cancel, resulting in equally radiative bonding and anti-bonding modes. The smallest positive gap width considered in our calculations $(3 \mathrm{~nm})$ is well within the validity range of simulation techniques based on purely classical electrodynamics. For more strongly coupled metallic nanoparticles, the use of a quantum-corrected approach would be required to take into account electron tunneling through the gap which limits optical field confinement. ${ }^{29}$

Zero gap corresponds to the 'kissing' point where the rounded endcaps of the two nanorods touch to form a single conducting contact point. ${ }^{22,23}$ The conducting bridge is associated with the appearance of a redshifted charge-transfer plasmon, and a reshaping of the mode spectrum. Upon further overlapping of the nanorods, the charge-transfer mode blueshifts due to the combined effects of increasing bridge inductance and a reduced effective antenna length. We approach the situation of the conductive L-shaped antenna for a gap of $-120 \mathrm{~nm}$. For even more negative gaps, another limit of a symmetric cross-antenna is reached. Here the mode splitting between bonding and anti-bonding completely disappears. We did not consider the regime beyond the L-shaped antenna in our experiments. In addition to traditional bonding and anti-bonding modes, Figure $2 \mathrm{~b}$ shows the appearance of additional modes on the blue side of the bonding mode for partially overlapping nanorods. Charge density maps (not shown) confirm that these modes are associated with a charge accumulation at the corner of the nanostructure. The presence of these additional 'corner' 
modes slightly modifies the polarization conversion spectra, resulting in small deviations from the simple two-oscillator model.

Figure $3 \mathrm{a}$ and $\mathrm{b}$ show results extracted from the numerical simulations of orthogonal dimer antennas with gap width varying between $-120 \mathrm{~nm}$ (L-shaped antenna) and $+145 \mathrm{~nm}$ (weakly coupled nanorod dimer). Figure $3 \mathrm{a}$ shows both the total and the Y-polarized scattered intensity at the wavelength $\lambda_{\mathrm{PC}}$ where the polarization conversion efficiency is highest. We define $\lambda_{\mathrm{PC}}$ as the wavelength at which the Y-polarized scattering intensity normalized to the total scattering intensity is maximum. Figure $3 \mathrm{~b}$ gives the spectral resonance positions for the anti-bonding $\left(\lambda_{A}\right)$ and bonding $\left(\lambda_{B}\right)$ modes, and the wavelength of maximum polarization conversion $\left(\lambda_{\mathrm{PC}}\right)$. The large change in the mode spectrum is observed around the kissing point of the two nanorods at zero gap. This transition is associated with a sharp decrease of the intensity scattered at the polarization conversion point.

To explain this behavior, we combined the information of Figures $3 a$ and $b$ to plot the scattering intensity at $\lambda_{\mathrm{PC}}$ against the dimensionless splitting parameter $\Delta \omega / \omega_{\text {ave }}$ in which $\omega_{\text {ave }}$ is the average of the angular frequencies of modes A and B. Figure $3 \mathrm{c}$ shows that, when plotted this way, the data of Figure 3a collapses onto a universal curve which covers both the capacitively and conductively coupled antenna regimes. The illustrations in Figures $3 \mathrm{a}$ and c indicate the positions of the different antenna geometries on this curve; clearly, the kissing arrangement provides the largest spectral splitting and is therefore located at the end of the range around $\Delta \omega / \omega_{\text {ave }} \sim 0.8$, while the fully connected L-shape and the capacitively coupled antennas seamlessly connect into a continuous curve with a transition around $\Delta \omega / \omega_{\text {ave }} \sim 0.35$. The point closest to zero splitting corresponds to the $448 \mathrm{~nm}$ gap antenna in which the nanorods are virtually uncoupled.

The total scattered intensity $\left(\sigma_{\mathrm{XX}}+\sigma_{\mathrm{XY}}\right)$ at $\lambda_{\mathrm{PC}}$ shows a monotonous decrease with increasing spectral splitting. This can be understood from the fact that with increasing spectral splitting, 
the overlap of the resonances is reduced and the antenna cross section at the wavelength $\lambda_{\mathrm{PC}}$, located in between the two resonance peaks, goes down. At the same time, the crosspolarized scattered intensity reaches a maximum for a relative spectral splitting around $\Delta \omega / \omega_{\text {ave }} \approx 0.2$. This maximum corresponds to an intermediate situation in which the two resonances are close enough so that efficient excitation of both eigenmodes remains possible and separated enough to allow for the appropriate dephasing between the excited oscillations. Next to the absolute intensity, a second important figure for polarization conversion is the degree of polarization (DOP) obtained in the polarization conversion. Figure $3 \mathrm{~d}$ shows the DOP, defined as the contrast between $\mathrm{X}$ and $\mathrm{Y}$ components of the scattering intensity:

$\mathrm{DOP}=\frac{\sigma_{X X}-\sigma_{X Y}}{\sigma_{X X}+\sigma_{X Y}}$

A DOP of 1 corresponds to pure $\mathrm{X}$ polarized radiation and no polarization conversion, while a DOP of -1 equals pure Y polarized radiation and $100 \%$ polarization conversion efficiency. The DOP in Figure 3d shows an increasing conversion efficiency with increasing spectral splitting, which can be understood from the convergence to exactly $\pi$ phase difference between modes A and B for large splitting (see Figure 1b).

The lines in Figure 3c and d represent the results from the simple two-oscillator model of eq 2 using optimized values for the width of the spectral modes. The model is tractable and closed-form expressions representing the model curves are presented in the Supporting Information Section S5. We clearly see that the numerical results follow the analytical model for all geometries under study, both in the capacitive and conductive regime. The curves are universal for the two oscillator model and scale with relative energy splittings and damping, as is further illustrated in the Supporting Information Figure S5. Markedly, the situation of the connected L-shape antenna, used in many of the experiments, corresponds to a point on the curve of around $\Delta \omega / \omega_{\text {ave }} \approx 0.4$ which neither produces the largest scattering intensity nor the highest conversion efficiency. 
Single-particle spectroscopy of symmetric and anti-symmetric modes. We make use of single-particle Spatial Modulation Spectroscopy (SMS) in reflection mode to obtain information on the scattering and polarization conversion of single plasmonic nanoantennas (see Methods and Supporting Information Section S1). ${ }^{30-32}$ The antennas of these studies were fabricated using e-beam lithography and consist of dimers of gold nanorods of fixed widths and heights of respectively $120 \mathrm{~nm}$ and $40 \mathrm{~nm}$, and with nanorod lengths $\mathrm{L}$ between $180 \mathrm{~nm}$ and $330 \mathrm{~nm}$. To illustrate the mode structure of capacitively coupled, orthogonal nanorod antennas, we measured first the 'pure' bonding and anti-bonding states of several representative $\mathrm{L}=230 \mathrm{~nm}$ antennas as shown in Scanning Electron Microscopy (SEM) images of Figure 4a,b. In this experiment the polarization was fixed to horizontal, while the antenna was azimuthally rotated to $+45^{\circ}$ and $-45^{\circ}$ with respect to this axis. Figure $4 a, b$ shows the experimental spectra for two antennas with large $(50 \mathrm{~nm})$ and small $(12 \mathrm{~nm})$ gaps. By exciting the antenna at $45^{\circ}$ with respect to the individual nanorods, it is possible to selectively address the bonding (B) and anti-bonding (A) modes. ${ }^{12,16}$ The corresponding calculated spectra are shown in Figure 4c,d while charge distributions for the two modes on the strongly coupled antenna are shown in Figure 4e,f. We see that the coupling more strongly affects the charges in the bonding mode, resulting in a large redshift of this mode for strongly coupled antennas. A mode splitting of $400 \mathrm{~nm}$, or $30 \%$, is found for the dimer antenna with $12 \mathrm{~nm}$ gap, which is in agreement with our numerical calculations. The experimentally measured modes are somewhat broader than the calculated modes, indicating some additional damping mechanisms in the experimental gold.

Linear polarization conversion. Following the identification of the hybridized modes, we investigated polarization conversion as a function of gap width for incident light polarized along the X-direction. Figure 5a-c show a selection of the experimental spectra for three different series of antennas with lengths $\mathrm{L}=180 \mathrm{~nm}$ (a), $230 \mathrm{~nm}$ (b) and $330 \mathrm{~nm}$ (c). The 
vertical units correspond to $10^{-13} \mathrm{~m}^{2}$ for the $\sigma_{\mathrm{XX}}$ extinction cross section; the $\sigma_{\mathrm{XY}}$ values were obtained using multiplication of the SMS signals with the experimental calibration factor of 5.0 (see Supporting Information Section S2). For the three different nanorod lengths, the antibonding mode was located at around $0.95 \mu \mathrm{m}(\mathrm{a}), 1.1 \mu \mathrm{m}$ (b) and $1.5 \mu \mathrm{m}(\mathrm{c})$. We note that the polarization conversion for the $12 \mathrm{~nm}$ gap antenna in Figure $5 \mathrm{~b}$ corresponds to the pure mode spectrum of Figure $4 \mathrm{~b}$. While the three antenna lengths showed qualitatively the same behaviour, the $\mathrm{L}=230 \mathrm{~nm}$ series of antennas produced the best results within the spectral range of our setup, providing an extensive range of splittings and polarization data. The scaling of energy splittings with antenna $\operatorname{size}^{33}$ means that smaller capacitive gaps are required for the $\mathrm{L}=180 \mathrm{~nm}$ antennas to observe any polarization rotation, while the bonding modes of the $\mathrm{L}=330 \mathrm{~nm}$ antennas are shifted into the infrared, outside of our experimentally accessible window. Very high polarization conversion efficiencies of up to $95 \%$ ( $D O P=-0.95)$ in the telecommunication range around $1.5 \mu \mathrm{m}$ is obtained for the $\mathrm{L}=330 \mathrm{~nm}$ antennas (see Supporting Information Figure S4c), however no information could be obtained on the corresponding mode splittings for these series, limiting further analysis in terms of universal scaling. Therefore in the following, we focus on the analysis for the $\mathrm{L}=230 \mathrm{~nm}$ antenna series. The very small gap width of $5 \mathrm{~nm}$ in Figure $5 \mathrm{a}$ was obtained using helium ion beam milling $^{32}$ to demonstrate the effect of energy splitting also for the shorter antenna series.

For large gaps, the mode splitting in Figure $5 \mathrm{~b}$ is small and only a small fraction of intensity is radiated by the antennas along the Y-polarization. With increasing coupling strength, the Y-polarized component increases until it exceeds the X-polarized component. This occurs around the critical point where a conducting bridge is formed. At the kissing point we observe a strong shift of the symmetric mode resonance toward longer wavelengths around 2 $\mu \mathrm{m}$. 
The spectrally resolved DOP is shown in Figure 6a,b for both the Green Dyadic Method calculations (a) and the experiment (b) for the $\mathrm{L}=230 \mathrm{~nm}$ length antennas (experimental results for the other antennas are shown in Supporting Information Figure S4). We find that the most efficient conversion takes place for antennas which are conductively coupled in the kissing regime, with a gap of around $-25 \mathrm{~nm}$. The spectral position of the minimum DOP (i.e. maximum of Y-polarized conversion $\lambda_{\mathrm{PC}}$ ) is plotted in Figure $6 \mathrm{c}$ together with the spectral resonance positions $\lambda_{\mathrm{A}}$ and $\lambda_{\mathrm{B}}$. Good agreement is obtained between the experimental results (symbols) and the numerical calculations (squares/lines). The combined information on spectral splittings and the DOP allows us to experimentally validate the universal scaling model described above and its conclusions. Figure $6 \mathrm{~d}$ shows the scaling of the DOP with mode splitting. The results confirm that stronger polarization conversion is obtained for larger mode splittings. We note that it is not possible to spectrally resolve splittings much smaller than the resonance width, resulting in some uncertainty in the results for $\Delta \omega / \omega_{\text {ave }}$ below 0.1 .

Linear to circular polarization conversion. As predicted using our analytical model, broadband polarization conversion between linear and circular polarization states exploiting plasmonic antennas is possible using orthogonal dimer antennas. Such linear-to-circular polarization conversion is of interest for ultracompact waveplates and optical biosensors. ${ }^{26,27}$ Our numerical simulations confirm that linear to circular polarization conversion is obtained around the wavelength where both antenna modes are $\pi / 2$ out of phase and when $\sigma_{\mathrm{xx}}$ and $\sigma_{\mathrm{xy}}$ are equal. This occurs at a relative splitting of $\Delta \omega / \omega_{\text {ave }} \approx 0.22$. In practice, approximate conditions can be found for a wider range of splittings. Indeed, we found good conditions for circular polarization conversion for the $\mathrm{L}=230 \mathrm{~nm},-52 \mathrm{~nm}$ gap antenna of Figure $5 \mathrm{~b}$, which has a spectral splitting $\Delta \omega / \omega_{\text {ave }}$ of around 0.5. In Figure 7 we present results for the linear to circular polarization conversion for this antenna (see Methods). Figure 7a shows the LCP and 
RCP scattering intensities emitted by the antenna, for linear input polarization along X. From these spectra, we calculated the degree of circular polarization (DOCP) analogous to the case for linear polarization, $\mathrm{DOCP}=\left(\sigma_{\mathrm{LCP}}-\sigma_{\mathrm{RCP}}\right) /\left(\sigma_{\mathrm{LCP}}+\sigma_{\mathrm{RCP}}\right)$. Indeed, we find a near perfect circularly polarized output (i.e., DOCP of 1) around $1.15 \mu \mathrm{m}$ wavelength.

To illustrate the validity of the simple two-oscillator model in case of linear to circular polarization conversion, we plot in Figure $7 \mathrm{c}$ and $\mathrm{d}$ the corresponding results obtained using eqs $4 \mathrm{a}$ and $4 \mathrm{~b}$. Here the relative widths and amplitudes of the two polarizabilities $\alpha_{a}$ and $\alpha_{b}$ were adjusted to match the experimental resonances. Qualitative agreement was obtained for the spectral response. In particular, we observe in both cases a Fano-type dip in the RCP spectrum where the perfect conversion takes place. Also both the model and experiment agree in the spectral position of the maximum CPL conversion. Additional oscillations are present in the experimental data which are not predicted by the simple two-oscillator model. These effects are attributed to contributions from the corner modes as observed in the numerical calculations of Figure $2 b$. The experimental results of Figure 7 demonstrate the feasibility of linear to circular polarization conversion in orthogonal nanorod antennas.

Our results confirm that polarization conversion caused by orthogonal antenna modes show a universal scaling with the mode splitting. Depending on the figure of merit chosen, the sweet spot for the largest absolute scattering efficiency is obtained for a relative splitting of 0.2 , which in our system is achieved in the capacitive coupling regime for gap width of around 10 $\mathrm{nm}$. The conversion efficiency monotonously increases with frequency splitting between the two modes, which is the highest for 'kissing' antennas where a narrow conductive bridge is formed. We emphasize that the fully connected L-shaped antennas used in many studies do not show the largest spectral splittings nor the strongest scattering intensity. The particular interest of the kissing regime has been previously identified in the chiral response of threedimensional plasmonic oligomers. ${ }^{34}$ The large spectral splitting and presence of higher order 
modes was found to facilitate a broadband circular dichroism of such structures. Similarly, we find in Figure 2 that the most broadband polarization conversion is obtained for the structures with the largest splitting, corresponding to the regime of touching nanorods.

A related, but different studies was presented by Yin et al., who studied similar arrangements of capacitively coupled perpendicular nanorods but with a displacement in the propagation direction. ${ }^{35}$ Such structures showed large optical chirality for D- and L-enantiomers, where for the D-enantiomer, right-hand circular polarized light coupled to the antibonding mode A and left-hand circular polarized light coupled to the antibonding mode B (and the reverse for the L-enantiomer). In our case, the structures have no separation along the propagation direction so our structures are both $\mathrm{D}$ - and L-enantiomers and both $\mathrm{A}$ and $\mathrm{B}$ modes are equally excited on the nanostructures for right-hand and left-hand circular polarized light.

The two-oscillator model gives fundamental bounds to the absolute degree and relative efficiency of polarization conversion that can be achieved in a simple coupled-oscillator system. Further improvement may be achieved exploiting e.g. coupling of antennas to a reflecting plane with multiple reflections, ${ }^{36}$ or all-dielectric antenna designs. ${ }^{37}$

\section{Conclusion}

In conclusion, we have investigated theoretically and experimentally the polarization conversion in both connected and disconnected gold nanorod dimer antennas. Our results demonstrate that the polarization conversion efficiency, scattered intensity and degree of polarization of both conductively or capacitively coupled dimer antennas depends on the spectral splitting between two orthogonal plasmon eigenmodes. By carefully designing the antenna morphology it is therefore possible to accurately control the degree of polarization of the scattered wave. In particular, we show that fully connected antennas used in metasurfaces are not optimized for conversion efficiency. Much higher efficiencies may be obtained using 
kissing antennas, if the reduced absolute scattering cross section can be compensated, e.g. by increasing the metasurface packing density or increasing the overall antenna scattering cross section. More complex planar architectures may be found using the simple design principles which combine efficient manipulation of the phase and polarization of optical waves, enabling new types of ultracompact optical devices.

\section{Methods}

3D-Green's Dyadic method. The Green's Dyadic method relies on a volume discretization of the system and allows to describe nanostructures of arbitrary morphology. ${ }^{28}$ The method rigorously takes into account the dielectric response of the substrate. The antennas were modelled as 3-dimensional objects with a constant thickness of 40nm and an in-plane projection consisting of a rectangular rod with two rounded endcaps (cf. Figure 4e,f). In the model, we simulated both illumination and collection of intensities over a numerical aperture (N.A.) of 0.5 , corresponding to the experimental configuration. The illumination polarization was taken along the $\mathrm{X}$-direction. For the detected light, both the $\mathrm{X}$ - and Y-polarized scattering intensities were calculated, corresponding to $\sigma_{\mathrm{XX}}$ and $\sigma_{\mathrm{XY}}$. To compute $\sigma_{\mathrm{XX}}$ and $\sigma_{\mathrm{XY}}$, we have calculated the electric field radiated in the far-field by the antennas when they are excited by an incident wave linearly polarized along $X$. The calculation of $\sigma_{X X}$ and $\sigma_{X Y}$ takes into account the numerical aperture of the collecting optics.

Nanoantenna fabrication. Nanoantennas were fabricated using electron beam lithography (JEOL 9300FS) at an accelerating voltage of $100 \mathrm{kV}$ and a writing current of $1 \mathrm{nA}$. The borofloat glass substrate was coated with a MMA (250 nm) / PMMA (150 nm) bilayer resist. A $20 \mathrm{~nm}$ thick conducting copolymer layer (Espacer 300Z) was used to prevent charging during electron beam exposure. After exposure the resist was developed in methyl isobutyl ketone (MIBK) : isopropyl alcohol (IPA) $1: 1$ for 60s. A $2 \mathrm{~nm} \mathrm{Cr}$ and $30 \mathrm{~nm}$ thick Au film 
were evaporated at a pressure of $5 \times 10^{-6}$ mbar. The lift-off was done manually by soaking in N-Methyl-2-pyrrolidone (NMP) for 20 mins.

Spatial Modulation Spectroscopy (SMS) is a technique suitable for extracting quantitative cross sections by the periodic modulation of the particle in a tightly focused laser beam. ${ }^{30,31}$ An achromatic Cassegrain reflective objective was used to cover an extended spectral range in the near-infrared. ${ }^{32}$ The Supporting Information Figure S1 presents the experimental arrangement in detail. A broadband supercontinuum light source was spectrally filtered and used as a tunable light source. The polarization state was defined using a polarization filter. Light reflected from the same surface was collected and analyzed using a second polarizer. While SMS has advantages compared to other techniques such as darkfield, it requires a careful calibration to apply the technique for polarization conversion studies. In particular, in order to compare the pure scattered intensities of the antenna, we need to take into account the contribution from direct (specular) reflection when P1 and P2 were taken parallel. The Supporting Information Figure S2 explains the calibration procedure, which involved measuring the spectra from a parallel dimer antenna, and which resulted in an overall multiplication factor of 5.0 for the cross-polarized configuration. A linear correction factor is justified since the extinction and scattered powers show the same quadratic dependence on antenna dipole moment in the limit of strong scattering [38]. This correction was independently verified using darkfield scattering measurements as shown in Supporting Information Figure S3.

Linear to circular polarization conversion. To quantify the conversion from a linear $\mathrm{X}$ polarized input to circular polarized output, two quarter waveplates (QWPs) were placed in the setup. QWP1 was placed in the input beam before the beam splitter and was aligned to prepare a circular state after the beam splitter. This state was converted back into linear Xpolarization using the second QWP2 which was placed just before the objective and oriented 
at $45^{\circ}$. The same QWP2 was used to convert the LCP and RCP circular polarized output states of the antenna to the $\mathrm{X}$ and $\mathrm{Y}$ linear polarizations, which could subsequently be analyzed using polarizer P2.

\section{AUTHOR INFORMATION}

\section{Corresponding Author}

*O.Muskens@ @ soton.ac.uk; * arnaud.arbouet@ @emes.fr

\section{Author Contributions}

The manuscript was written through contributions of all authors. All authors have given approval to the final version of the manuscript.

The authors would like to thank Dr Christian Girard for fruitful discussions. This work was supported by the computing facility center CALMIP of the University Paul Sabatier of Toulouse France. This work was supported by DSTL and DGA through a joint UK-France PhD studentship, and by EPSRC through grant EP/J011797/1. OM acknowledges support through an EPSRC fellowship EP/J016918/1.

\section{Supporting Information}

Description and schematic overview of experimental setup, calibration procedure for spatial modulation microscopy, comparison with results from darkfield spectroscopy, experimental degree of polarization versus gap width for different antenna lengths, and analytical expressions from the two-oscillator model. This material is available free of charge via the Internet at http://pubs.acs.org.

\section{REFERENCES}

1. Li, Z.; Utlu, M.; Ozbay, E. Chiral Metamaterials: From Optical Activity And Negative Refractive Index To Asymmetric Transmission J. Opt. 2013, 15, 023001. 
2. Soukoulis, C. M.; Wegener, M. Past Achievements And Future Challenges In The Development Of Three-Dimensional Photonic Metamaterials Nat. Photon. 2013, 5, 523-530.

3. Yu, N.; Genevet, P.; Kats, M.; Aieta, F.; Tetienne, J.-P.; Capasso, F.; Gaburro, Z. Light Propagation with Phase Discontinuities: Generalized Laws of Reflection and Refraction Science 2011, 334, 333-337.

4. Ni, X.; Ishii, S.; Kildishev, A. V.; Shalaev, V. M. Ultra-Thin, Planar, Babinet-Inverted Plasmonic Metalenses Light: Sc. \& Appl. 2013, 2, e7

5. Huang, L.; Chen, X.; Mühlenbernd, H.; Zhang, H.; Chen, S.; Bai, B.; Tan, Q.; Jin, G.; Cheah, K.-W.; Qiu, C. W. et al. Three-Dimensional Optical Holography Using A Plasmonic Metasurface Nat. Comm. 2013, 4, 2808.

6. Ni, X.; Emani, N. K.; Kildishev, A. V.; Boltasseva, A.;Shalaev, V. M. Broadband Light Bending With Plasmonic Nanoantennas Science 2012, 335, 427.

7. Sun, S; He, Q.; Xiao, S.; Hu, Q.; Zhou, L. Gradient-Index Meta-Surfaces As A Bridge Linking Propagating Waves And Surface Waves Nat. Mater. 2012, 11, 426-431.

8. Huang, L.; Chen, X.; Mühlenbernd, H.; Li, G.; Bai, B.; Tan, Q.; Jin, G.; Zentgraf, T.; Zhang, S. Dispersionless Phase Discontinuities For Controlling Light Propagation Nano Lett. 2012, 12, 5750-5755.

9. Sun, S.; Yang, K.-Y.; Wang, C.-M.; Juan, T.-K.; Chen, W. T.; Liao, C. Y.; He, Q.; Xiao, S.; Kung, W.-T.; Guo, G.-Y. et al. High-Efficiency Broadband Anomalous Reflection By Gradient Meta-Surfaces Nano Lett. 2012, 12, 6223- 6229;

10. Chen, W. T.; Yang, K. Y.; Wang, C. M.; Huang, Y. W.; Sun, G.; Chiang, I. D.; Liao, C. Y.; Hsu, W. L.; Lin, H. T.; Tsai, D. P., High-Efficiency Broadband Meta-Hologram with Polarization-Controlled Dual Images, Nano Lett. 2014, 14, 225- 230.

11. Sukharev, M.; Sung, J.; Spears, K. G.; Seideman, T. Optical Properties Of Metal Nanoparticles With No Center Of Inversion Symmetry: Observation Of Volume Plasmons Phys. Rev. B 2007, 76, 184302.

12. Sung, J.; Sukharev, M.; Hicks, E. M.; Duyne, R. P. Van; Seideman, T.; Spears, K. G. Nanoparticle Spectroscopy: Birefringence In Two-Dimensional Arrays Of L-Shaped Silver Nanoparticles J. Phys. Chem. C 2008, 3252-3260.

13. Jing, Y.; Jia-Sen, Z.; Xiao-Fei W.; Qi-Huang, G. Resonant Modes Of L-Shaped Gold Nanoparticles Chinese Phys. Lett. 2009, 26, 067802.

14. Husu, H.; Mäkitalo, J.; Laukkanen, J.; Kuittinen, M; Kauranen, M. Particle Plasmon Resonances In L-Shaped Gold Nanoparticles Opt. Expr. 2010, 18, 16601-16606.

15. Yang, J.; Zhang, J. Subwavelength Quarter-Waveplate Composed Of L-Shaped Metal Nanoparticles Plasmonics 2011, 6, 251-254.

16. Kats, M.; Genevet, P.; Aoust, G.; Yu, N.; Blanchard, R.; Aieta, F.; Gaburro, Z.; Capasso, F. Giant Birefringence In Optical Antenna Arrays With Widely Tailorable Optical Anisotropy Proc. Natl. Acad. Sci. 2012, 109, 12364-12368. 
17. Vercruysse, D.; Sonnefraud, Y.; Verellen, N.; Fuchs, F. B.; Di Martino, G.; Lagae, L.; Moshchalkov, V. V; Maier, S. A.; Van Dorpe, P. Unidirectional Side Scattering Of Light By A Single-Element Nanoantenna Nano Lett. 2013, 13, 3843-3849.

18. Kauranen, M.; Zayats, A. V. Nonlinear Plasmonics Nat. Photon. 2012, 6, 737-748.

19. Panaro, S.; Toma, A.; Proietti Zaccaria, R.; Chirumamilla, M.; Saeed, a.; Razzari, L.; Das, G.; Liberale, C.; De Angelis, F.; Di Fabrizio, E. Design And Top-Down Fabrication Of Metallic L-Shape Gap Nanoantennas Supporting Plasmon-Polariton Modes Microelectron. Eng. 2013, 111, 91-95.

20. Funston, A. M.; Novo, C.; Davis, T. J.; Mulvaney, P. Plasmon Coupling of Gold Nanorods at Short Distances and in Different Geometries Nano Lett. 2009, 9, 16511658 .

21. Shao L., Woo K. C., Chen H., Jin Z., Wang J., Lin H. Q. Angle- And Energy-Resolved Plasmon Coupling In Gold Nanorod Dimers ACS Nano. 2010, 4, 3053-3062.

22. Romero, I.; Aizpurua, J.; Bryant, G. W.; García de Abajo, F. J. Plasmons In Nearly Touching Metallic Nanoparticles: Singular Response In The Limit Of Touching Dimers Opt. Expr. 2006, 14, 9988-9999

23. Halas, N. J.; Lal, S.; Chang, W.-S.; Link, S.; Nordlander, P. Plasmons In Strongly Coupled Metallic Nanostructures Chem Rev. 2011, 111, 3913-3961.

24. Acimovic, S. S.; Kreuzer, M. P.; González, M. U.; Quidant, R. Plasmon Near-Field Coupling In Metal Dimers As A Step Toward Single-Molecule Sensing ACS Nano 2009, 3, 1231-1237.

25. Luk'yanchuk, B.; Zheludev, N. I.; Maier, S. A.; Halas, N. J.; Nordlander, P.; Giessen, H.; Chong, C. T. The Fano Resonance In Plasmonic Nanostructures And Metamaterials Nat. Mater. 2010, 9, 707-715.

26. Zhao, Y.; Alu, A. Tailoring The Dispersion Of Plasmonic Nanorods To Realize Broadband Optical Meta-Waveplates Nano Lett. 2013, 13, 1086-1091.

27. Abasahl, B.; Dutta-Gupta, S.; Santschi, C.; Martin, O. J. F. Coupling Strength Can Control the Polarization Twist of a Plasmonic Antenna Nano Lett. 2013, 13, 45754579.

28. Girard, C.; Dujardin, E.; Baffou, G.; Quidant, R. Shaping And Manipulation Of Light Fields With Bottom-Up Plasmonic Structures New J. Phys. 2008, 10, 105016.

29. Savage, K. J.; Hawkeye, M. M.; Esteban, R.; Borisov, A. G.; Aizpurua, J.; Baumberg, J. J. Revealing The Quantum Regime In Tunnelling Plasmonics Nature 2012, 491, 574-577.

30. Arbouet, A.; Christofilos, D.; Fatti, N. Del; Valle, F.; Huntzinger, J. R.; Arnaud, L.; Billaud, P.; Broyer, M. Direct Measurement of the Single-Metal-Cluster Optical Absorption Phys. Rev. Lett. 2004, 93, 127401.

31. Muskens, O.; Billaud, P.; Broyer, M.; Del Fatti, N.; Vallée, F. Optical Extinction Spectrum of a Single Metal Nanoparticle: Quantitative Characterization of a Particle and of Its Local Environment Phys. Rev. B 2008, 78, 205410. 
32. Wang, Y.; Abb, M.; Boden, S.; Aizpurua, J.; de Groot, C. H.; Muskens, O. L. Ultrafast Nonlinear Control of Progressively Loaded, Single Plasmonic Nanoantennas Fabricated Using Helium Ion Milling Nano Lett. 2013, 13, 5647-5653.

33. Woo K,C; Shao L.; Chen H.; Liang Y.; Wang J., Lin H. Q. Universal Scaling And Fano Resonance In The Plasmon Coupling Between Gold Nanorods ACS Nano. 2011, 5, 5976-5986.

34. Hentschel, M; Wu, L.; Schäferling, M.; Bai, P.; Li E. P., Giessen, H. Optical Properties Of Chiral Three-Dimensional Plasmonic Oligomers At The Onset Of Charge-Transfer Plasmons ACS Nano 2012, 6, 10355-10365.

35. Yin, X.; Schäferling, M.; Metzger, B.; Giessen H. Interpreting Chiral Nanophotonic Spectra: The Plasmonic Born-Kuhn Model Nano Lett. 2013, 13, 6238-6243.

36. Pors A.; Nielsen M. G .; Eriksen R. L .; Bozhevolnyi S. I. Broadband Focusing Flat Mirrors Based On Plasmonic Gradient Metasurfaces Nano Lett. 2013, 13, 829-834.

37. Yang, Y.; Wang, W.; Moitra, P.; Kravchenko, I.,; Briggs, D. P.; Valentine, J. Dielectric Meta-Reflectarray for Broadband Linear Polarization Conversion and Optical Vortex Generation Nano Lett. 2014, 14, 1394-1399.

38. Le Ru, E. C.; Somerville, W. R. C.; Auguié, B. Radiative Correction In Approximate Treatments Of Electromagnetic Scattering By Point And Body Scatterers Phys. Rev. A 2013, 87, 012504.

\section{FIGURES}
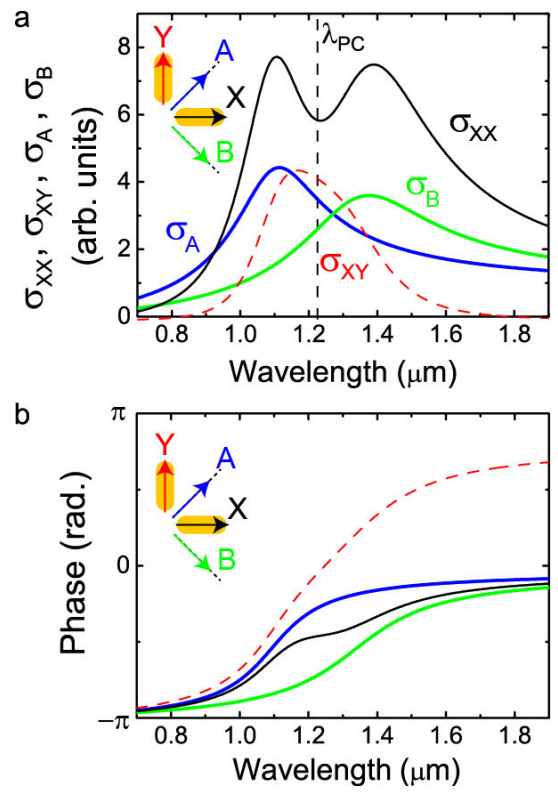

Figure 1 a Scattering intensities for incident light polarized along diagonals $\mathbf{A}\left(\sigma_{A}\right)$ and $\mathbf{B}\left(\sigma_{B}\right)$ of the antenna structure, and intensities of scattered light along $X\left(\sigma_{X X}\right)$ and $Y\left(\sigma_{X Y}\right)$, both for input polarization along $X$. Inset shows directions of $\mathbf{A}, \mathbf{B}, \mathbf{X}, \mathbf{Y}$, with respect to the dimer antenna (yellow rods). The wavelength of maximum linear polarization conversion is denoted by $\lambda_{\mathrm{PC}}$. $\mathrm{b}$ Phases of the antenna dipole moment $\mathbf{P}$ for same conditions as a, from analytical model. 

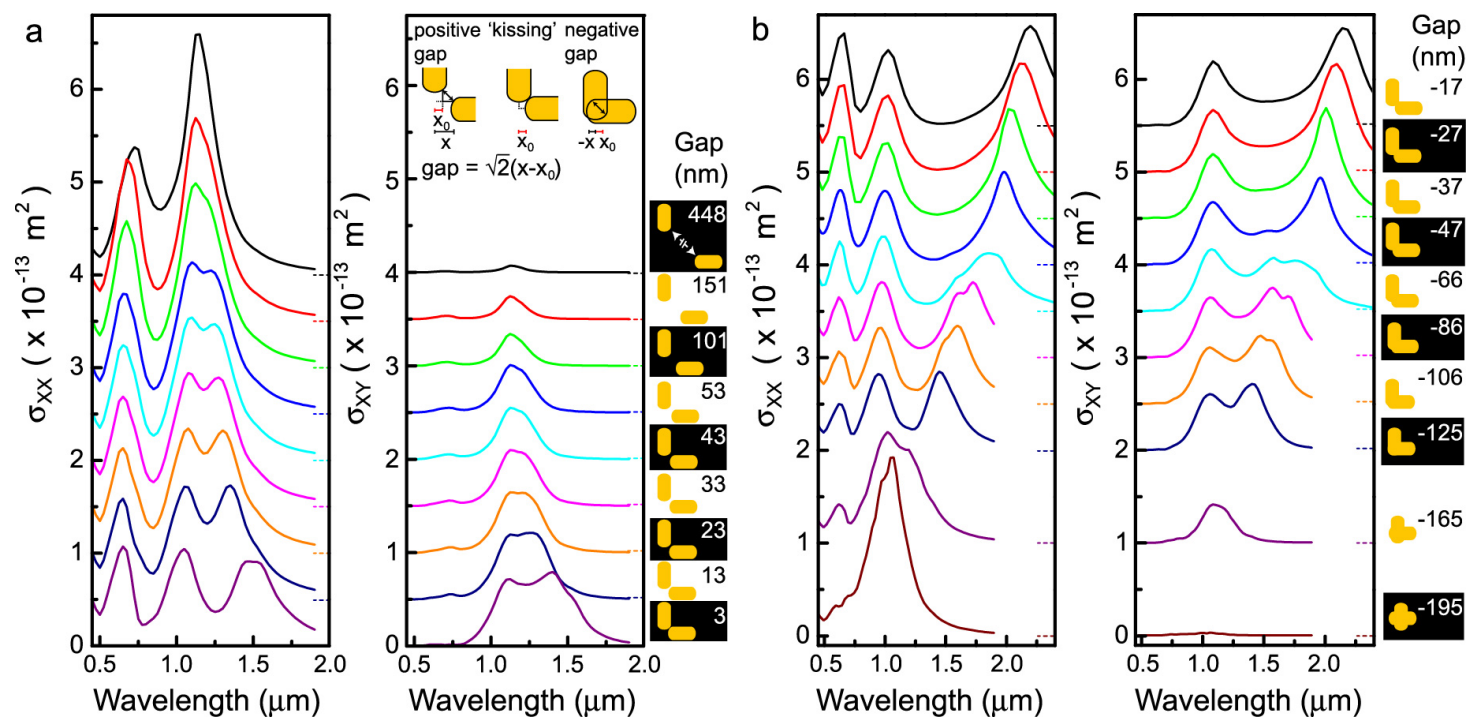

Figure 2 Calculated collected scattering intensities $\sigma_{\mathrm{XX}}$ and $\sigma_{\mathrm{XY}}$ against gap width, for capacitively coupled antennas (a) and conductively coupled antennas (b) for $\mathrm{L}=\mathbf{2 3 0} \mathrm{nm}$ nanorod lengths. Curves are vertically offset as indicated by dashed lines. Schemes on right of graphs correspond to antenna geometries with corresponding gap values. Inset: definition of gap parameter for capacitive and conductive antennas. The offset $x_{0}$ is defined from the kissing point where the antennas form a singular conducting contact.
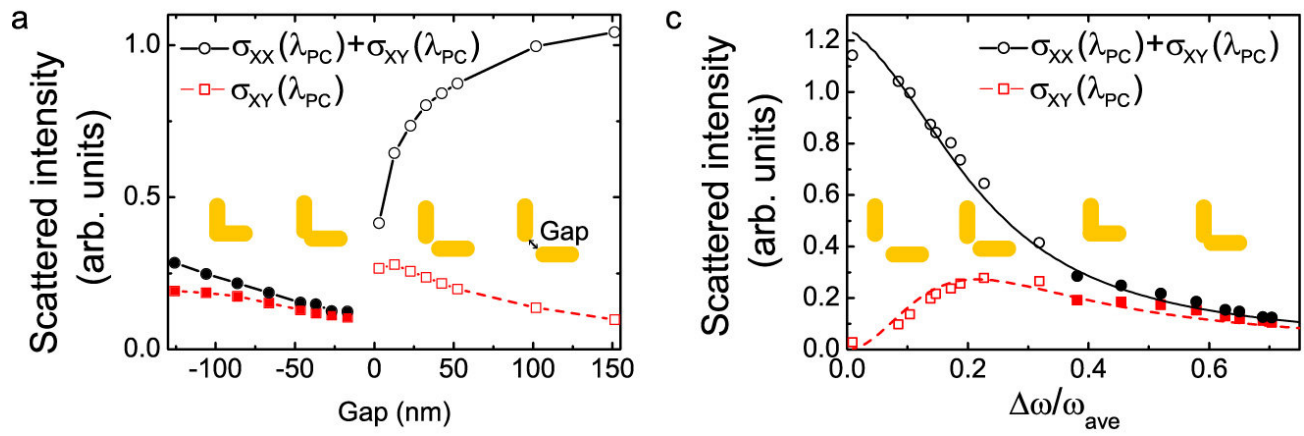

b

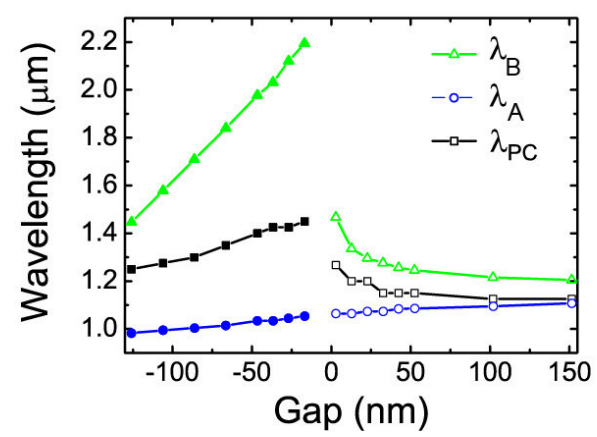

d

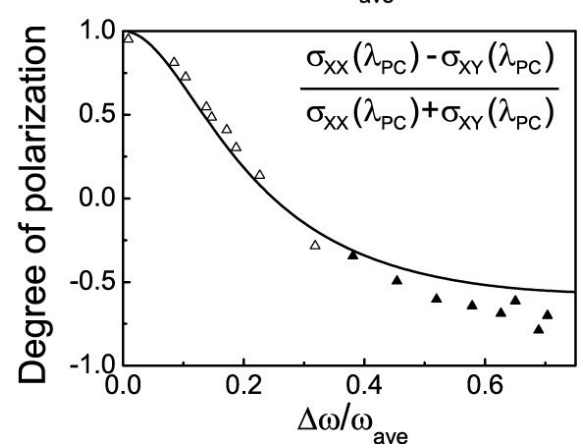

Figure 3a,b Results from 3D Green Dyadic Method simulations (symbols). a Total scattered intensity (black dots) and Y-polarized component (red squares) at maximum conversion wavelength $\lambda_{\mathrm{PC}}$, for input polarization along $\mathbf{X}$. b Spectral positions of modes $\mathrm{A}$ and $\mathrm{B}$, and $\lambda_{\mathrm{PC}}$ versus gap width. $\mathrm{c}$ Same as a but plotted against relative frequency splitting $\Delta \omega / \omega_{\text {ave }}$. d Degree of polarization at maximum conversion wavelength (eq 6) against relative frequency splitting. Closed symbols in a-d: conductively coupled, open symbols: capacitively coupled antennas. Lines in c, $d$ are results from analytical two-oscillator model (respectively Supporting Information eqs S8-S9 and S4-S5 for c and d). 

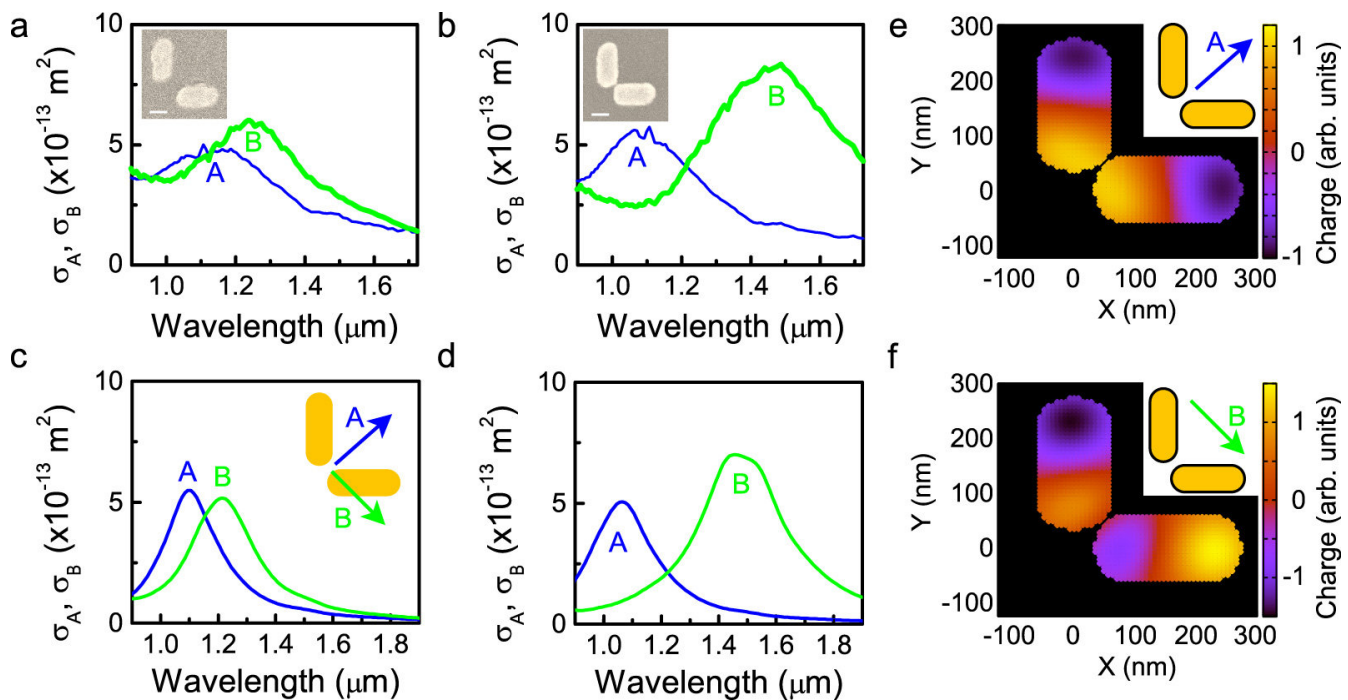

Figure 4 Experimental a,b and calculated c,d spectra for $\mathrm{L}=\mathbf{2 3 0} \mathrm{nm}$ dimer antennas with large (54 $\mathrm{nm})$ and small (12 $\mathrm{nm})$ gaps for polarizations corresponding to the orientations A and B as shown in the inset of c. Insets: SEM images, scale bars, $100 \mathrm{~nm}$. e, f Charge distributions in the antennas corresponding to the anti-bonding (A) and bonding (B) dipole modes at $1.1 \mu \mathrm{m}$ and $1.5 \mu \mathrm{m}$ wavelength, respectively.
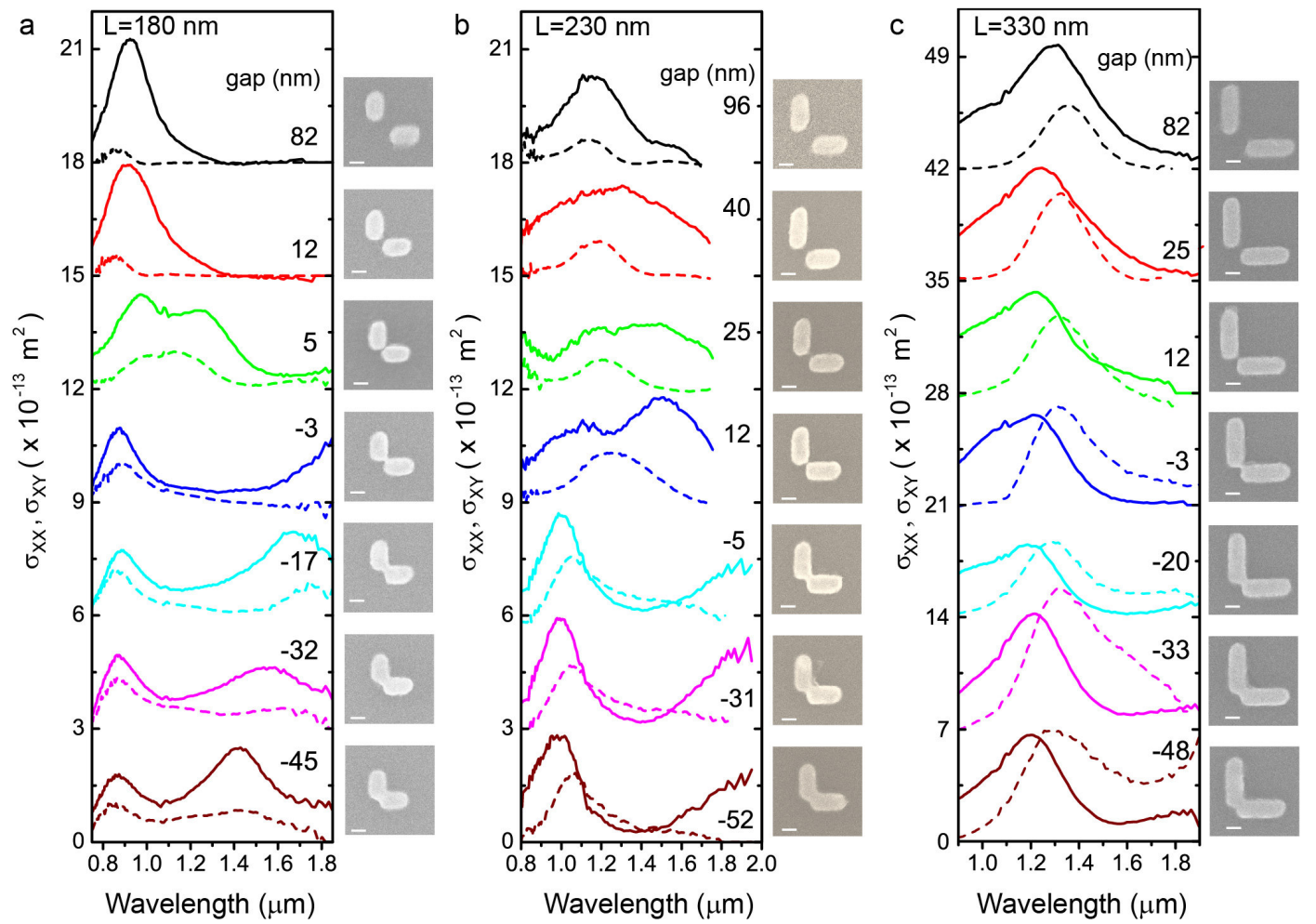

Figure 5 a-c Experimental spectra of $L=180 \mathrm{~nm}(a), L=230 \mathrm{~nm}$ (b) and $\mathrm{L}=330 \mathrm{~nm}$ (c) orthogonal dimer antennas with gaps from $96 \mathrm{~nm}$ to $-52 \mathrm{~nm}$. Solid lines: intensity for X-polarized component $\left(\sigma_{\mathrm{XX}}\right)$ of scattered light, dashed lines: intensity for $\mathrm{Y}$-polarized component $\left(\sigma_{\mathrm{XY}}\right)$, both for input polarization along $\mathrm{X}$. Curves are vertically offset by $3 \times 10^{-13}$ $\mathrm{m}^{2}$ for a,b and by $7 \times 10^{-13} \mathrm{~m}^{2}$ for c. Insets: SEM images, scale bars, $100 \mathrm{~nm}$. 
a

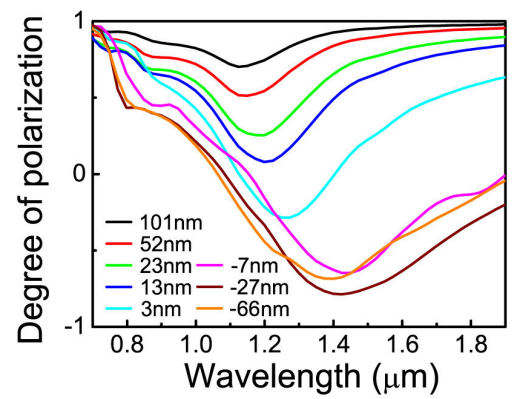

C

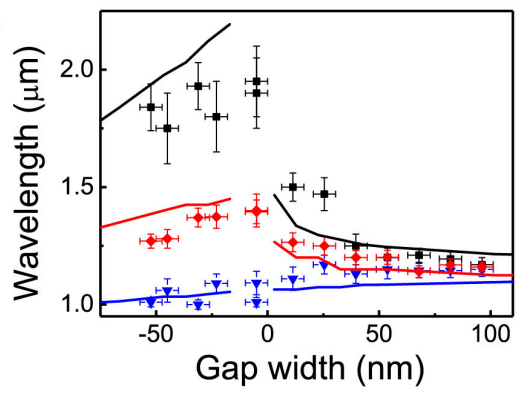

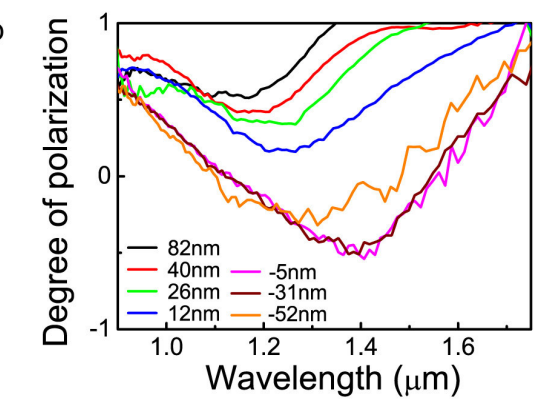

d

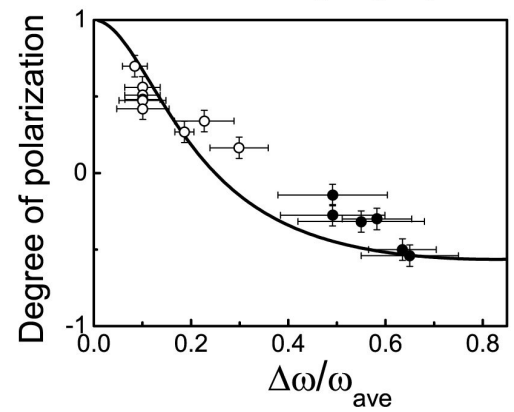

Figure 6 Degree of polarization (eq 6 ) for different antenna spacings for $\mathrm{L}=\mathbf{2 3 0} \mathrm{nm}$ orthogonal dimers of Figure $5 \mathrm{~b}$, calculated (a) and experimental (b). c Experimental resonance positions of bonding (squares, black) and anti-bonding (triangles, blue) modes, and position of maximal polarization conversion (dots, red), with results from numerical simulations using the 3D-Green Dyadic Method (lines). d Degree of polarization versus frequency splitting, for capacitively (open circles) and conductively (closed dots) coupled antennas. Line corresponds to result from twooscillator model (cf. Fig. 3d).
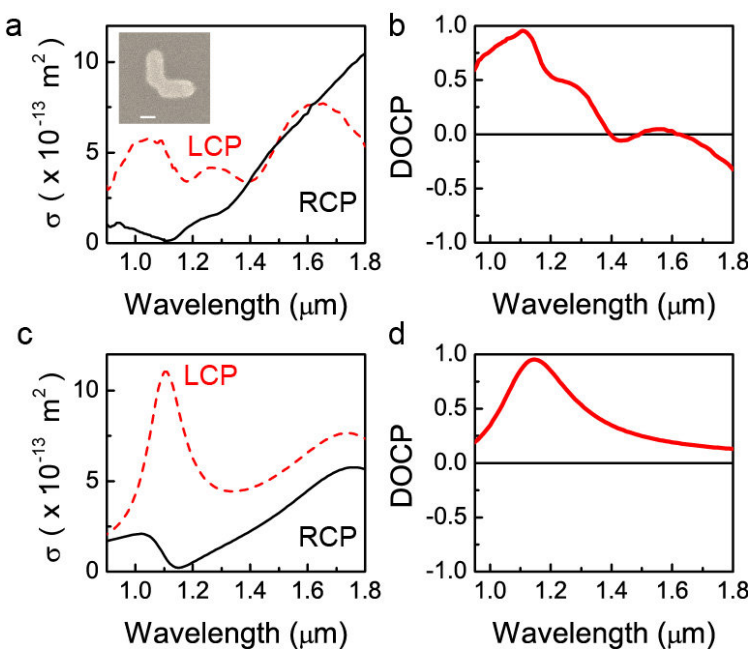

Figure 7 a Experimental scattering intensity $\sigma$ for left-hand (LCP) and right-hand (RCP) circularly polarized components of antenna radiation, obtained using $\mathrm{L}=\mathbf{2 3 0} \mathrm{nm}, \mathbf{- 5 2} \mathrm{nm}$ gap antenna of Figure 5b. Inset: SEM image, scale bar $100 \mathrm{~nm}$. b Degree of Circular Polarization (DOCP) obtained from a. c,d Circularly polarized scattering (c) and DOCP (d) calculated using two-oscillator model with spectral positions, widths and amplitudes corresponding to experiment. Both experiment and model show near-perfect circular polarization (DOCP=1) around $1.15 \mu \mathrm{m}$ wavelength. 

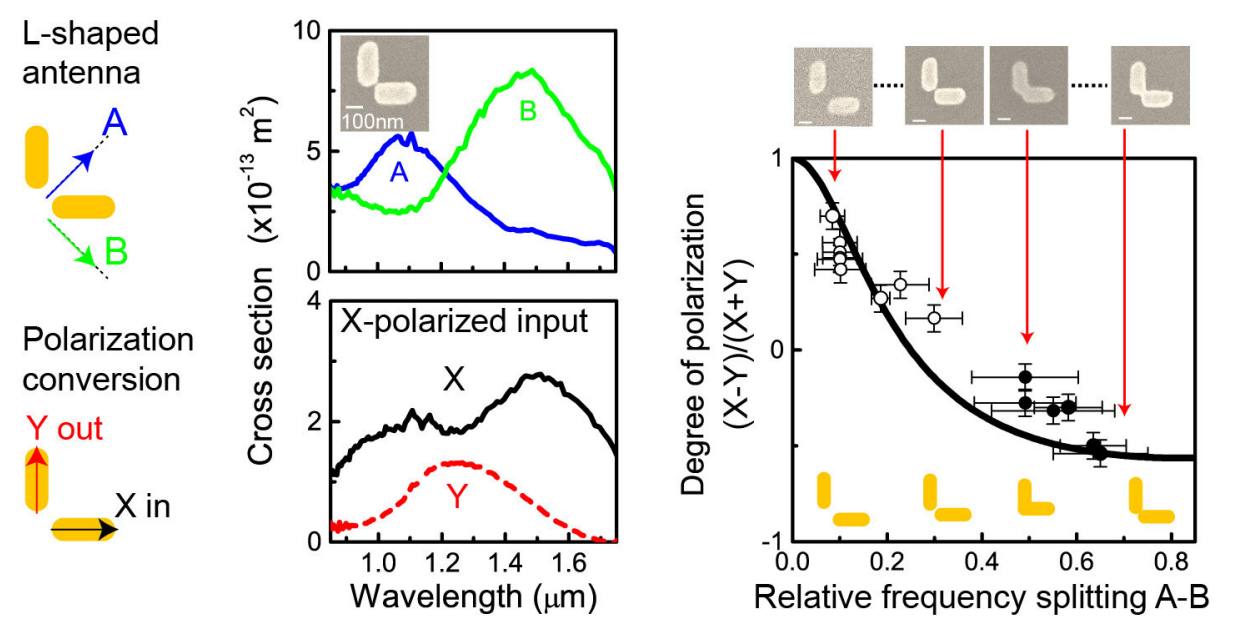

Table of Contents figure 